\title{
Uso del tiempo libre, jóvenes y delito en México
}

\section{Use of leisure-time, youth and crime in Mexico}

\author{
Nelson Arteaga* \\ Cecilia Gayet* \\ Alejandro Alegría*
}

\begin{abstract}
The article discusses that the risk of youth in Mexico to be victims of a crime during their leisure time depends on their activities inside or outside home as well as their lifestyles. Also the relation between these elements is mediated by demographic and socioeconomic variables. This means that leisure activities are related to the position of the victim in the social structure. So, not only the activities of the leisure time determine the processes of victimization but also those who perform them.
\end{abstract}

Keywords: victimization, leisure time, security, youth.

\section{Resumen}

El artículo muestra que en México la exposición de los jóvenes a ser víctimas de un delito durante el uso de su tiempo libre depende de las actividades que realizan dentro o fuera del hogar, así como de sus estilos de vida; esta relación se encuentra mediada por variables sociodemográficas y adscripciones socioeconómicas. Las actividades en el tiempo libre se vinculan con la posición que ocupa la víctima en la estructura social, esto es, no son sólo las actividades que se realizan en el tiempo libre las que potencialmente definen los procesos de victimización, sino también quiénes las realizan.

Palabras clave: victimización, tiempo libre, seguridad, jóvenes.

* Facultad Latinoamericana de Ciencias Sociales (Flacso), México. Correos-e: nelson.arteaga@ flacso.edu.mx, cgayet@flacso.edu.mx y aalegria@flacso.edu.mx 


\section{Introducción}

El incremento del sentimiento de inseguridad en México ha contribuido en parte al cierre del espacio público. La proliferación de zonas habitacionales cerradas, algunas de ellas vigiladas permanente, al igual que la video vigilancia de espacios dedicados al ocio y la diversión dan cuenta de cómo el espacio público se ha convertido para muchos en un lugar peligroso que debe evitarse en la medida de lo posible (Valenzuela, 2013).

Para ciertos sectores sociales, las calles son de hecho un campo de batalla donde las autoridades son incapaces de restaurar el orden; en algunos casos, incluso, se responsabiliza a las propias autoridades de seguridad de propiciar la violencia. ${ }^{1}$ En este contexto ganan terreno los discursos que consideran prudente evitar los espacios fuera del hogar, salvo para realizar actividades labores, escolares y comerciales, particularmente en centros comerciales fortificados (Jorquera, 2011), lo anterior mina la confianza social en la medida en que se valoran como válidos para la convivencia, los espacios de interacción que se encuentran regulados por algún mecanismo de supervisión y control (Salmi et al., 2007).

Uno de los principales destinatarios de estos discursos son los jóvenes ${ }^{2}$ pues, en la medida en que se les considera un sector vulnerable en contextos de inseguridad, se juzga pertinente mantenerlos dentro del hogar y "fuera de las calles" como estrategia de protección. Se argumenta que más allá de las actividades laborales o escolares, los jóvenes deben permanecer en casa; también se recomienda que sus traslados -ya sea a través del transporte público o privado-se hagan en compañía de otras personas (por ejemplo, padres, tutores o pares).

Se pondera como positivo que las actividades de ocio y uso del tiempo libre se lleven a cabo en espacios que cuenten con mecanismos de monitoreo, como centros comerciales o calles que se sabe están bajo la supervisión de sistemas de video vigilancia. El impulso de estas estrategias de protección ha adquirido cierta legitimidad social, a tal grado que se diseñan medidas de política pública en este sentido. Por ejemplo, se han establecido toques de queda para jóvenes menores de edad en municipios como Tecate, Baja California; Ciudad Juárez, Chihuahua; Zongolica, Veracruz; Tlalnepantla, Estado de México; Nadadores, Coahuila y Acuña, Coahuila. Sin embargo, habría que preguntarse si efectivamente, y en qué

${ }^{1}$ Las ciudades más representativas de esta imagen de la calle como campo de batalla son Monterrey, Matamoros, Reynosa y Ciudad Juárez, donde grupos de narcotraficantes han bloqueado las avenidas para enfrentar a la policía y al ejército.

${ }^{2}$ Una encuesta realizada en el 2013 por México Unido Contra la Delincuencia y Mitofsky, senala que $44 \%$ de la población ha prohibido a sus hijos salir a la calle a jugar, y $47 \%$ ha dejado de salir en la noche (Mexico Unido contra la Delincuencia, 2013). 
medida, resguardarse en el hogar es o no una estrategia que protege a los jóvenes de su posible victimización.

La sociología criminal ha venido apuntando, sobre todo a partir de los años sesenta, a que la victimización en el sector de los jóvenes responde más a ciertos estilos de vida, comportamientos rutinarios y desplazamientos construidos desde cierta cotidianidad, que al hecho de ser joven (Stein, 2010; Wilcox et al., 2003; Cohen y Felson, 1979; Hindelang et al., 1978).

Ciertamente, esos comportamientos y estilos de vida están relacionados con el perfil demográfico y socioeconómico de las víctimas y requieren ser tomados en cuenta. Una parte importante de estas reflexiones tuvo como referente las corrientes interaccionistas o microsociológicas, que ponían en el centro del análisis el peso que tienen las rutinas en la construcción de escenarios de vulnerabilidad -al volver invisibles los posibles riesgos y amenazas circundantes- y protección -al proporcionar a las personas un cierto control de su entorno-. La victimización quedó ligada entonces a la capacidad de las personas para identificar y sortear potenciales situaciones de peligro en función de marcos valorativos específicos (Walklate, 1998). ${ }^{3}$

Una de las aportaciones más significativas de estos trabajos fue advertir que la prevención de la victimización no necesariamente dependía de la presencia de las fuerzas del orden o cualquier otra autoridad, sino de capacidades cognitivas socialmente adquiridas para identificar y ponderar, en una situación específica, escenarios de riesgo (Collins, 2008). De esta manera, se entiende que las personas garantizan su protección gracias a la habilidad y conocimiento que tienen para leer su entorno, decodificarlo y plantear con ello una posición estratégica de defensa frente al riesgo.

Así, un entorno inseguro en el que impera el crimen y la violencia no significa un ambiente caótico y sin sentido, sino que expresa la mayoría de las veces un orden social inteligible para quienes viven en él (Anderson, 2000). Leer este orden es una capacidad que se aprehende y que permite a las personas continuar con sus vidas, pese a la presencia de escenarios de alto riesgo (Lupton 1999).

Por tanto, evitar actividades fuera del hogar puede ser una medida de protección en el corto plazo, pero con el tiempo limita la capacidad de las personas para moverse en los contextos sociales que se desarrollan más allá del entorno aparentemente protector del hogar. Si esto es así,

\footnotetext{
${ }^{3}$ Efectivamente, la policía desempeña un papel central en la determinación de la seguridad. No obstante en tanto la policía no está siempre en todos lados y en todo momento, las personas tienden a generar sus propios mecanismos de protección frente a potenciales escenarios delictivos (González, 2002; Blancas-Madrigal, 2004; Job, 2013; Chávez et al. 2004; Zócalo-Saltillo, 2012; La policiaca, 2011).
} 
apelar a que los jóvenes se queden en casa para desarrollar sus actividades de tiempo libre y ocio, podría estar dilatando la posibilidad de que desenvuelvan experiencias y procesos de interacción, que, en última instancia, contribuyen a generar mecanismos de autoprotección. Ciertamente la calle no es en sí misma una fuente de experiencias positivas y constructivas; sin embargo, es un espacio en el que se puede aprender a negociar con los riesgos que son inherentes a él.

El presente documento tiene por objetivo poner a prueba este supuesto. Por una parte, indaga si el hecho de que los jóvenes realicen durante la mayor parte del tiempo libre actividades en espacios domésticos -ver televisión, jugar videojuegos o leer- reduce las posibilidades de ser víctimas de delito en comparación con aquellos que prefieren actividades fuera del hogar-como ir al parque, a bailar o salir de compras-. Y en el caso contrario, se plantea si aquéllos que acostumbran a salir más, tienen menores probabilidades de ser víctimas de delitos por contar con mejores habilidades para definir las condiciones de riesgo. Este artículo investiga sobre la relación entre las actividades de ocio de los jóvenes y el hecho de convertirse en víctimas de la inseguridad. ${ }^{4}$

El texto parte de la distinción entre actividades dentro del hogar y fuera de hogar, y no entre espacio privado y público, por el hecho de que esta última diferenciación resulta inadecuada frente a la presencia de espacios donde la frontera entre privado y público se ha desdibujado (Richards, 2013). Están, por un lado, las llamadas propiedades privadas de masas -aeropuertos, estaciones de tren o metro- en las que se ofrecen distintas opciones de descanso, trabajo y movilidad a amplios sectores de la población, pero funcionan como espacios privados (Wakefield, 2005). Están por el otro lado los espacios "públicos-privados" (Carmona, 2010) que aparentan ser públicos -como los grandes centros comerciales, cines, grandes cafeterías, discotecas y bares-, pero son realmente privados (Nieto et al., 2002).

Cada uno de estos espacios establece formas de gestión de la seguridad y de la población muy distintas, donde además conviven distintos planos de ejercicio y aplicación de la ley, por ejemplo, policías privadas y públicas e incluso semi-públicas o semi-privadas. En este sentido, tratar de distinguir las actividades de ocio que los jóvenes realizan en su tiempo libre por su adscripción a lo privado o lo público oculta la compleja variedad de los espacios. Por el contrario, distinguir entre las actividades de

${ }^{4} \mathrm{El}$ artículo no busca explorar las causas de la violencia y el crimen entre jóvenes. Algunos trabajos en América Latina han realizado indagaciones interesantes en este sentido, ligando los procesos de empobrecimiento, crisis económica, la reconfiguración del núcleo familiar, escolar y laboral, entre otros factores, a la victimización de los jóvenes. Para una revisión del estado del arte sobre estas aproximaciones en América Latina ver a Imbusch et al. (2011). 
ocio que los jóvenes realizan en su tiempo libre en función del lugar donde las desarrollan, ya sea donde habitan o en aquellos espacios externos al hogar, establece un marco definido en términos de un adentro y un afuera que permite contrastar dos mundos que, en el ámbito de la construcción social de la inseguridad, se consideran tradicionalmente como opuestos. 5

El artículo se divide en cuatro partes: en la primera se desarrollan algunos conceptos básicos que permiten comprender el peso de los procesos de interacción y las rutinas sociales en la definición de escenarios de riesgo y protección. Se busca con ello establecer un marco general de análisis para observar en qué medida las actividades que los jóvenes realizan en su tiempo libre, ya sea dentro o fuera del hogar, influye en su victimización. En la segunda parte se expone la metodología que se desarrolló para probar esta relación. Se consideran los límites y alcances de la fuente de datos que se utilizó, así como los presupuestos del análisis. En la tercera sección se analizan y comentan los resultados. Finalmente, en el apartado de conclusiones, se discuten las implicaciones de los resultados pero sobre todo se sugiere la necesidad de ampliar y reforzar las fuentes de información con el fin de comprender las dinámicas de victimización de los jóvenes.

\section{Código de la calle, seguridad y rutinas en contextos de riesgo}

Las corrientes ligadas a cierta perspectiva microinteraccionista han señalado de forma recurrente que la victimización se encuentra vinculada a la capacidad de los sujetos para interpretar qué está pasando en ciertos contextos y situaciones. Inspirados particularmente en los trabajos de Goffman (1986) y Garfinkel (1984), en línea con las reflexiones de Simmel (2001), estas perspectivas destacan cómo los sujetos y grupos sociales interpretan el espacio social y, a partir de ello, definen una determinada posición y una fachada social; ambas permiten, entre otras cosas, ponerse a resguardo de situaciones que sitúan en riesgo la propiedad y la integridad física de las personas.

Sutherland (1988) mostró de forma muy temprana que los delincuentes - particularmente en su análisis de estafadores y carteristas- despliegan estrategias similares de decodificación de las situaciones con el fin de adoptar una fachada "inofensiva" para ocultar sus intenciones frente a sus potenciales víctimas. ${ }^{6}$ En trabajos más recientes, Anderson (2000) ha

\footnotetext{
${ }^{5}$ Por otro lado, cabe mencionar que la información de la encuesta con la que se trabaja en este artículo no permite definir con precisión la diversidad de "espacios públicos" en el que realizan los jóvenes sus actividades de tiempo libre. Pero sí permite hacer supuestos sobre actividades que se llevan a cabo dentro y fuera del hogar.

${ }^{6}$ En este sentido se podría decir que existen rutinas que ponen a los sujetos en condición de ser víctimas de un delito. Los delincuentes serían de esta forma "expertos" en decodificar dichas rutinas
} 
señalado que en contextos de alta criminalidad y violencia delictiva las personas aprenden a negociar las condiciones de peligro en espacios abiertos. Esto implica reconocer lo que este autor denomina el código de la calle: un conjunto de prescripciones y proscripciones -o reglas informales de comportamiento organizado- que permite reconocer las situaciones que pueden transformar a una persona en el objetivo de un acto criminal. Para este autor, el código de la calle emerge de forma más clara cuando no hay presencia de la policía -o ésta es prácticamente inexistente-, lo que obliga a las personas a desplegar sus habilidades de reconocimiento del entorno para hacerse de las adecuadas garantías de seguridad y protección.

Esta capacidad de comprender el código de la calle es una habilidad que se aprende en el día a día de la vida. Como toda habilidad, no sólo es una experiencia individual, sino colectiva, que permite la construcción de relaciones sociales estables y de confianza, aún pese a la presencia de un claro contexto de incertidumbre (Sennett, 2012). Las habilidades para decodificar el entorno implican la puesta en marcha de una serie de estrategias de convivencia cristalizadas en mecanismos de comunicación que transmiten códigos y símbolos específicos sobre cómo comportarse en una situación particular.

El sentimiento de inseguridad en un vecindario, calle o plaza depende, precisamente, de la comprensión del código de la calle. Esto explica, en parte, por qué no todas las personas y grupos sociales consideran un espacio determinado como seguro o inseguro (Heitgerd y Bursik, 1987). Incluso, cuando los medios de comunicación tipifican un lugar como de alto riesgo -señalando que sus habitantes viven en constante miedo- lo cierto es que se puede encontrar que eso no es así para quienes habitan en ellos (Walklate, 1998).

Como sugiere Giddens (1997), los individuos tienden a desarrollar un marco de seguridad cuando logran consolidar un número determinado de rutinas. La gente hace frente a los peligros a partir de fórmulas emocionales y de comportamientos que se convierten en parte de su vida cotidiana. Dichas fórmulas resultan medios de protección frente al peligro que los otros pudieran representar y son la substancia básica de las interacciones cotidianas que normalmente no percibimos.

Goffman (1977) describió este proceso a partir de lo que llamó la "indiferencia civil": la manera en que las personas se miran en el espacio de la interacción de una forma subrepticia y controlada, desapercibida, pero que tiene como objetivo comprobar que las otras personas no representan una amenaza; dicha indiferencia civil se enmarca en lo que este

en las que los sujetos se mueven de forma tal que sus movimientos resultan previsibles y, por tanto, vulnerables (Stein, 2010). Para un análisis más contemporáneo sobre cómo los delincuentes toman sus decisiones con respecto al tipo de víctima y delito es posible consultar a Jacobs y Wright, 2010. 
mismo autor denomina como los rituales de tacto y confianza. Estos marcos de seguridad hacen posible que las personas puedan definir su mundo de vida -que se trasladen a trabajar, estudiar o divertirse- pese a la presencia de un clima de inseguridad (Walklate, 2001; Blokland, 2008).

Cuando una persona es víctima de un delito se fractura la seguridad que ha construido, cuestiona su conocimiento del código de la calle y altera sus rutinas (Mawby y Walklate, 1997). Con el tiempo, sin embargo, las personas caen en cuenta de que su victimización resultaba muy difícil de evitar (Coaffe et al., 2009), por lo que regresan poco a poco a sus rutinas, sumando la experiencia vivida al script del código de la calle y recuperando de esta manera algo de su seguridad perdida.

Algunos estudios muestran que, conforme se asimila la victimización, las personas atribuyen lo sucedido a un "descuido" o a una falta de atención al entorno (Shapland y Hall, 2007). El que una víctima restablezca su seguridad depende, ciertamente, del tipo de delito que sufrió; algunos estudios señalan que la asimilación del delito puede llevar entre dos semanas y dos meses, para el caso de delitos menores; mucho más tiempo si la víctima sufrió un delito grave, por ejemplo: violación, robo con violencia o secuestro (Denkers y Winkel, 1998; Davis y Friedman, 1985; Resick, 1987). Este "regreso a la normalidad" sugiere que la victimización es un proceso en el que la víctima somete su experiencia a una constante interpretación que le permite convivir con sus efectos (Walklate, 2011). Esto por supuesto, como señalan Threadgold y Nilan (2009), se encuentra mediado en función de la adscripción socioeconómica a la que pertenece la víctima.

El uso de espacios abiertos es central, por tanto, para hacerse de las habilidades que permiten leer el código de la calle, adquirir una cierta seguridad y enmarcar las experiencias de victimización. Desafortunadamente, en contextos marcados por un alto sentimiento de inseguridad, se pondera como positiva la restricción del uso del espacio fuera del hogar: se cree en parte que esto garantiza la seguridad de las personas y reduce significativamente las tasas de victimización.?

Resalta además que la calle se transforma en un sinónimo de riesgo cuando se trata de proteger a niños y jóvenes de la criminalidad. Si bien por un lado se reconoce que algunas actividades que los jóvenes realizan fuera de casa son inevitables, como ir al trabajo o la escuela, se considera que éstas deben llevarse a cabo con la mayor de las atenciones y cuidado. Las actividades en el tiempo libre, por el otro lado, son calificadas como

7 La expansión de vecindarios y centros comerciales cerrados es una tendencia generalizada a escala global que muestra los procesos de "bunkerización” de espacios públicos y privados (Caldeira, 2000), esto reafirma la sensación de miedo y riesgo que representan los espacios "abiertos" sin controles de monitoreo, como arcos para la detección de metales o cámaras de video vigilancia. 
lúdicas y por tanto se cree que, pese a todas las precauciones, su práctica lleva al descuido, la desatención y el desdén por mantener los cuidados que protegen contra los riesgos potenciales del entorno.

Finalmente, otras opiniones que se emiten desde el mundo de los adultos sugieren que la juventud es una etapa en la que predominan las actividades de riesgo y se soslayan los cuidados sobre los peligros del entorno (Green y Singleton, 2006). En este sentido, no resulta extraño que se incrementen los llamados a restringir sus actividades de tiempo libre fuera del hogar.

Si bien algunos estudios muestran que las actividades realizadas en el tiempo libre fuera del hogar son condicionantes para ser víctima de un delito, no hay un patrón definido de dicha relación (Stein, 2010; ésta se encuentra mediada por variables demográficas -edad y sexo, por ejemplo-, estructurales - grado de urbanización, ingreso por hogar-y estilos de vida. Distintos análisis multinivel han mostrado que cuando estas variables se toman en consideración se obtienen distintos grados de relación entre ser víctima de un crimen y las actividades de ocio fuera del hogar.

Wilsem et al. (2003) encuentran, por ejemplo, una relación significativa entre las actividades que las personas realizan, incluyendo las laborales y de tiempo libre, con la victimización cuando se incluyen variables estructurales. Sin embargo, Tseloni y Farell (2002) establecen que esta relación no es significativa cuando se incluyen variables sociodemográficas. En tanto que Miethe y Drass (1999) consideran que tiene un peso mayor el estilo de vida, por ejemplo, cuando las actividades de tiempo libre se llevan a cabo en la noche. Algunas de estas variaciones en los resultados responden a la forma en que se han caracterizado y clasificado las actividades de ocio, trabajo, e incluso los propios delitos. Si la observación se lleva a cabo sobre un grupo particular, como los jóvenes, esto se complejiza sobre todo por la variedad de los rangos que se utilizan para definir a este grupo de población.

Pese a las diferencias en resultados, estos estudios permiten aproximarse a la forma en que se relacionan las actividades de tiempo libre y la victimización entre los jóvenes. En un contexto de inseguridad como el que vive México, este tipo de trabajos permite plantear algunas hipótesis, aunque sea de forma incipiente. Cabe resaltar que la mayoría de los trabajos, según la interpretación de distintos autores (Valenzuela, 2002; Imbusch et al., 2011; Pérez et al., 2014) privilegian tres temas: cómo se involucran los jóvenes en actos o actividades delictivas, cómo son criminalizados por el sistema de seguridad y justicia, y en qué medida ciertos factores estructurales - marginación, exclusión y pobreza- son un factor que empuja a los jóvenes a involucrarse en el mundo del crimen. 
En este sentido, resulta relevante el trabajo que Camarena (2015) lleva a cabo utilizando los datos de la Encuesta Nacional de Victimización y Percepción sobre Seguridad Pública (Envipe) de 2014, porque aborda el tema de la sensación de inseguridad y miedo al delito entre los jóvenes en México. Muestra que sólo 11\% de los jóvenes del país no parece preocuparse por la inseguridad; a 8\% le preocupa la situación de inseguridad en el país, pero se siente seguro; en tanto que para 32\% de los jóvenes, la inseguridad no es una preocupación, aunque considera que puede ser víctima de ella; finalmente, $49 \%$ vincula la preocupación por la inseguridad con una posible victimización a futuro. Cuando Camarena (2015) analiza cuál es el entorno inmediato donde los jóvenes se sienten más inseguros determina que $42 \%$ considera su colonia; $63 \%$ que su municipio o delegación, en tanto que $73 \%$ su entidad federativa.

En este tenor, Camarena (2015) encuentra que $82 \%$ de los jóvenes se siente seguro en su casa, en contraste con $9 \%$ que percibe los espacios donde transita, trabaja o estudia como seguros. Para la investigación que presentamos, esta información resulta relevante porque confirma la percepción de que es más seguro estar en el hogar que fuera de él. Sin embargo, al no dejar claro en qué medida esta sensación de miedo e inseguridad se relaciona por el tipo de actividad que realizan, resulta difícil establecer el lugar de enunciación desde el que se construye dicha sensación.

Si bien el presente artículo no busca cubrir este vacío, su aportación estriba en establecer un primer parámetro para determinar en qué medida las actividades de ocio fuera o dentro del hogar que realizan los jóvenes facilitan o inhiben que sean víctimas de un delito. En este sentido, aporta información relevante para contribuir a la discusión sobre la supuesta protección que proporcionan las actividades que los jóvenes realizan en el hogar en escenarios de inseguridad.

\section{Metodología}

El trabajo tiene el objetivo de responder si el tipo de actividades de esparcimiento que realizan los jóvenes en México se relaciona con ser víctima de delito. Como se señaló en los apartados anteriores, la literatura plantea dos hipótesis contrarias: a) si los jóvenes realizan actividades dentro del hogar estarán menos expuestos a ser víctimas de delito; $b$ ) si realizan actividades fuera del hogar tendrán mejores capacidades para interpretar los riesgos del entorno y por tanto estarán menos expuestos a ser víctimas del delito. Para investigar si estas hipótesis tienen sustento empírico se ha tomado en consideración - tal como la literatura revisada sugiere- el tipo 
de actividades que se realizan dentro y fuera del hogar, así como variables demográficas, estructurales y de estilos de vida.

Se utiliza para ello la Encuesta Nacional de la Juventud del 2010 que incluye una pregunta para los jóvenes entrevistados sobre si han sido víctimas de delito en los doce meses previos, otra sobre qué tipo de delito (pueden responder más de uno), y una más sobre si presentaron denuncia ante las autoridades.

Además pregunta a los jóvenes cuál es la principal actividad que realizan para divertirse en el tiempo libre. Se presenta en primer lugar un análisis descriptivo y posteriormente se realizan modelos logísticos multinivel a fin de saber si existe relación entre ser víctima de delito y el tipo de actividades que declaran realizar en el tiempo libre (que refleja un cierto estilo de vida), controlado por variables demográficas (edad y sexo), estructurales (escolaridad, posesión de aparato de telefonía móvil), y en un segundo nivel, entidad federativa, a fin de poner a prueba las hipótesis mencionadas. De esta manera, se trató de establecer un vínculo entre las variables que se consideran necesarias para entender la victimización entre jóvenes -expuestas en el apartado anterior- y la información que proporciona la encuesta analizada.

Aunque si bien es cierto que este ajuste se encuentra condicionado por el tipo de preguntas planteadas en la encuesta -la cual fue diseñada con objetivos distintos a los de este trabajo- permite dar cuenta tanto de las actividades del tiempo libre con variables demográficas y estructurales, que son centrales para alcanzar los objetivos de esta investigación.

En este estudio, el supuesto que es necesario considerar, dadas las limitaciones de la fuente de datos, es que la principal actividad de diversión que los jóvenes dijeron realizar al momento de la encuesta es semejante a la que realizaron durante todo el año previo. Como se ha expuesto más arriba, este supuesto se sostiene a partir del hecho de que cuando una persona es víctima de un delito, inmediatamente después cambia sus hábitos, pero con el paso del tiempo regresa a sus actividades habituales y, de alguna manera, minimiza o procesa el episodio, incorporándolo como parte de su habilidad para leer el código de la calle. Esto depende, como ya se señaló, del tipo de crimen al que la persona se ha visto sometida (Shapland y Hall, 2007). Si es víctima de un delito menor, como un robo sin violencia, tiende a sobrepasar el momento más rápido que si fue víctima de una violación o un asalto con violencia.

Como se puede observar, de la información que se tiene de la Encuesta Nacional de la Juventud del 2010, la mayor prevalencia de delitos que sufren los jóvenes se encuadra en este tipo de delitos menores; son pocos en realidad los que han sufrido algún tipo de crimen violento como agresión, extorsión, violación o secuestro (cuadro 1). En este sentido se puede 
sostener el supuesto de que muy probablemente la principal actividad de diversión que los jóvenes dijeron realizar al momento de la encuesta es semejante a la que realizaron durante todo el año previo.

Para el estudio se seleccionó a jóvenes de 15 a 24 años de edad de ambos sexos. El número de casos es de 16,921, y con información completa es de 16,155 , se presentarán resultados ponderados. Se estableció que la entidad federativa fuera en un segundo nivel en el modelo logístico, ya que los jóvenes de una misma entidad federativa - pese a las posibles diferencias regionales, o entre espacios urbanos y rurales- tienen en ese espacio geográfico tendencialmente una misma dinámica demográfica, condiciones de estructura social semejantes, estilos de vida particulares, instituciones públicas similares - un mismo sistema judicial, policial y penal- $y$, por tanto, comparten ambientes de inseguridad similares, por lo que es conveniente controlar la correlación intra-estrato. Además, las pruebas estadísticas indican que sí es estadísticamente significativo el cambio entre modelos de un nivel y otros de dos niveles, por lo que resulta más apropiado realizar modelos de dos niveles.

\section{Resultados}

Las características generales de la población bajo estudio pueden verse en el cuadro 1. Entre los jóvenes entrevistados de 15 a 24 años, casi $10 \%$ dijo haber sido víctima de algún delito en los doce meses previos a la encuesta, con mayor reporte por parte de los hombres (cuadro 1), y del grupo de 20 a 24 años (gráfica 1). En cuanto al tipo de delito sufrido para este trabajo se considera aquél que señalaron como primera opción (la encuesta permite enumerar más de un tipo de delito). La mayoría señaló robo, seguido por agresión y extorsión que se presentaron en muy pequeñas proporciones y, aún menos, secuestros (cuadro 2). Un quinto de los varones y un cuarto de las mujeres que sufrieron algún delito dijo que había presentado la denuncia ante alguna autoridad (cuadro 1).

La gráfica 2 presenta los resultados del reporte de víctima de delito por entidad federativa. El Distrito Federal, Michoacán, Morelos, Baja California, Aguascalientes, Colima, Jalisco, Quintana Roo y Nuevo León se encuentran por encima de la media nacional. En general los hombres reportan en mayor proporción haber sufrido algún delito en el año previo, donde destaca el Distrito Federal, con $28 \%$ de los varones. Sin embargo, en algunas entidades federativas la proporción de mujeres que reportó delitos fue mayor a la de los hombres, es el caso de Nayarit, Baja California Sur, Puebla, Sinaloa, Yucatán y Estado de México. 


\section{Cuadro 1}

Características de la población entrevistada de 15 a 24 años, por sexo, porcentajes

\begin{tabular}{|c|c|c|c|}
\hline & \multicolumn{2}{|c|}{ Sexo } & \multirow[b]{2}{*}{$\begin{array}{c}\text { Total } \\
n=16,921\end{array}$} \\
\hline & $\begin{array}{l}\text { Hombre } \\
n=7964\end{array}$ & $\begin{array}{c}\text { Mujer } \\
n=8957\end{array}$ & \\
\hline \multicolumn{4}{|l|}{ Edad } \\
\hline 15 & 11,6 & 10,3 & 10,9 \\
\hline 16 & 11,1 & 10,9 & 11,0 \\
\hline 17 & 11,2 & 11,6 & 11,4 \\
\hline 18 & 15,0 & 12,8 & 13,9 \\
\hline 19 & 10,2 & 10,2 & 10,2 \\
\hline 20 & 10,0 & 9,0 & 9,5 \\
\hline 21 & 6,9 & 7,6 & 7,3 \\
\hline 22 & 7,8 & 8,7 & 8,2 \\
\hline 23 & 6,8 & 8,4 & 7,6 \\
\hline 24 & 9,4 & 10,5 & 10,0 \\
\hline Total & 100,0 & 100,0 & 100,0 \\
\hline \multicolumn{4}{|l|}{ Escolaridad } \\
\hline Primaria o menos & 7,8 & 8,9 & 8,3 \\
\hline Algún nivel de secundaria & 39,7 & 39,0 & 39,4 \\
\hline Algún nivel de preparatoria o equivalente & 37,3 & 37,7 & 37,5 \\
\hline Algún nivel de licenciatura o más & 15,2 & 14,4 & 14,8 \\
\hline Total & 100,0 & 100,0 & 100,0 \\
\hline \multicolumn{4}{|l|}{ Tenencia de celular } \\
\hline No & 32,7 & 31,4 & 32,0 \\
\hline Sí & 67,3 & 68,6 & 68,0 \\
\hline Total & 100,0 & 100,0 & 100,0 \\
\hline \multicolumn{4}{|c|}{ Principal actividad que realizan para divertirse en el tiempo libre } \\
\hline Ir al parque & 4,1 & 6,6 & 5,4 \\
\hline Ir al cine & 3,3 & 5,1 & 4,3 \\
\hline Ir a bailar & 4,2 & 6,2 & 5,2 \\
\hline Jugar videojuegos & 5,4 & 0,6 & 3,0 \\
\hline Ir de compras & 0,9 & 3,2 & 2,0 \\
\hline Hacer deporte & 19,2 & 4,8 & 11,9 \\
\hline Reunirme con amigos & 26,8 & 21,3 & 24,0 \\
\hline Salir con mi pareja & 10,3 & 14,0 & 12,1 \\
\hline Conectarte a internet & 3,8 & 5,0 & 4,4 \\
\hline Escuchar música & 7,4 & 12,0 & 9,8 \\
\hline Ver televisión & 8,9 & 13,1 & 11,0 \\
\hline Leer & 1,6 & 2,7 & 2,2 \\
\hline Otra & 2,6 & 3,6 & 3,1 \\
\hline No contestó & 1,5 & 1,8 & 1,6 \\
\hline Total & 100,0 & 100,0 & 100,0 \\
\hline \multicolumn{4}{|l|}{ Victima de delito } \\
\hline No & 88,7 & 92,3 & 90,5 \\
\hline Sí & 11,3 & 7,7 & 9,5 \\
\hline Total & 100,0 & 100,0 & 100,0 \\
\hline \multicolumn{4}{|l|}{ Denuncia ante autoridades por el delito sufrido } \\
\hline No ha sido víctima de delito & 88,7 & 92,3 & 90,5 \\
\hline Sí & 1,9 & 1,7 & 1,8 \\
\hline No & 9,4 & 6,0 & 7,7 \\
\hline \multirow{2}{*}{ No contestó } & 0,0 & 0,0 & 0,0 \\
\hline & 100,0 & 100,0 & 100,0 \\
\hline
\end{tabular}

Fuente: elaboración propia con base en la (IMJ, 2010). 


\section{Gráfica 1}

Proporción de jóvenes de 15 a 24 años como víctimas de delito por sexo y grupo de edad, porcentajes

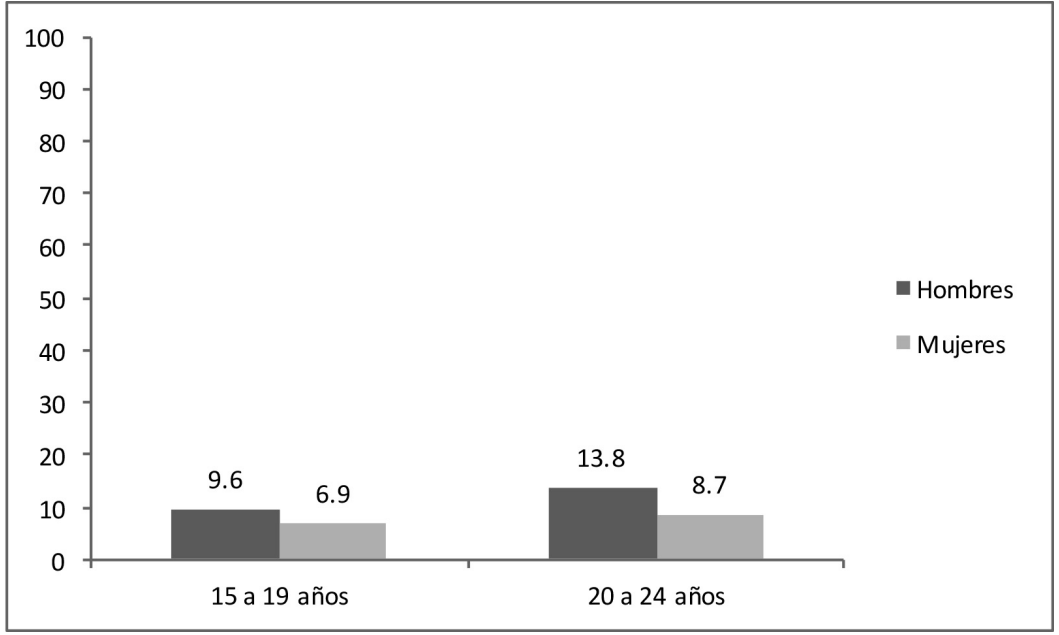

Fuente: elaboración propia con base en la Enjuve (IMJ, 2010).

\section{Cuadro 2}

Tipo de delito sufrido en los últimos doce meses (primera opción), por sexo y grupo de edad, porcentajes

\begin{tabular}{lcccccc}
\hline & \multicolumn{3}{c}{ De 15 a 19 $(n=9724)$} & \multicolumn{3}{c}{ De 20 a 24 (n=7197) } \\
& Total & Hombres & Mujeres & Total & Hombres & Mujeres \\
\hline $\begin{array}{l}\text { No ha sido víctima de } \\
\text { algún delito, durante }\end{array}$ & 91,7 & 90,4 & 93,1 & 88,9 & 86,2 & 91,3 \\
los últimos doce meses & & & & & & \\
Robo & 6,7 & 7,7 & 5,6 & 9,1 & 11,3 & 7,1 \\
Secuestro & 0,4 & 0,4 & 0,3 & 0,2 & 0,1 & 0,3 \\
Violación & 0 & 0 & 0,1 & 0 & 0 & 0 \\
Extorsión & 0,4 & 0,5 & 0,2 & 0,8 & 1,2 & 0,4 \\
Agresión & 0,6 & 0,8 & 0,5 & 0,8 & 0,8 & 0,7 \\
Otro & 0,1 & 0,1 & 0 & 0,1 & 0,2 & 0,1 \\
No contestó & 0,1 & 0,1 & 0,2 & 0,1 & 0,2 & 0,1 \\
Total & 100 & 100 & 100 & 100 & 100 & 100 \\
\hline
\end{tabular}

Fuente: elaboración propia con base en la Enjuve (IMJ, 2010). 


\section{Gráfica 2 \\ Proporción de jóvenes de 15 a 24 ańos que reportó haber sufrido algún delito en el ańo previo, por entidad federativa y sexo}

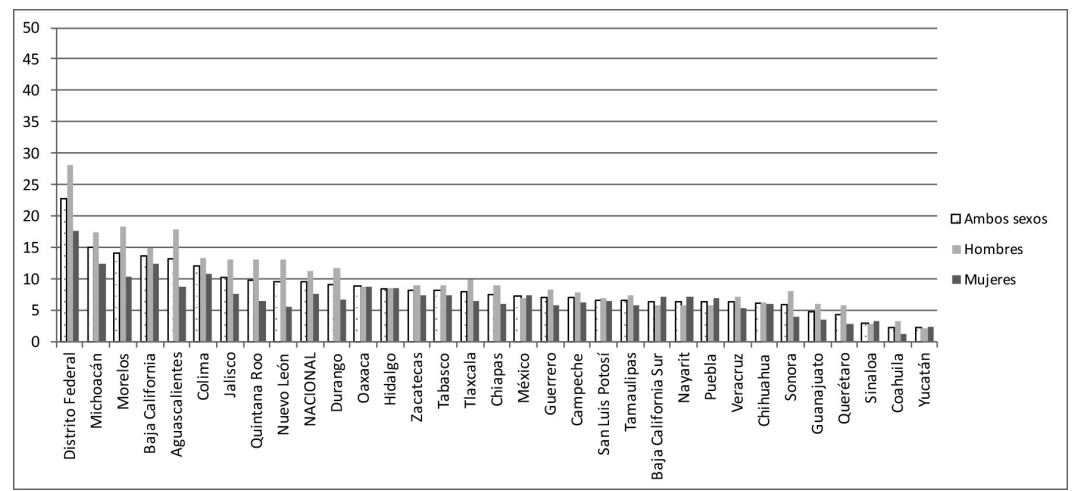

Fuente: elaboración propia con base en la Enjuve (IMJ, 2010).

Para poner en relación las variables de interés (ser víctima del delito y actividades de esparcimiento) se agruparon las actividades que figuran como opciones en la encuesta en dos categorías. La primera incluye las actividades que se realizan principalmente fuera del hogar (ir al parque, ir al cine, ir a bailar, ir de compras, hacer deporte, reunirme con amigos, salir con mi pareja) y, la segunda, las que usualmente se realizan al interior del hogar (jugar videojuegos, ver televisión, leer, conectarte a internet, escuchar música).

Un primer análisis descriptivo muestra que en todas las entidades federativas, los jóvenes realizan en mayor proporción actividades de esparcimiento fuera del hogar, tal como puede verse en la gráfica 3.

En cuanto a la relación de interés entre ser víctima del delito y principal actividad de esparcimiento (dentro o fuera del hogar), la gráfica 4 muestra la proporción de jóvenes que han sido víctimas de delito según el lugar de la actividad por entidad federativa.

A fin de poner a prueba las hipótesis sobre si las actividades de diversión fuera del hogar ponen a los jóvenes en mayor riesgo de sufrir delitos que las actividades dentro del hogar, se estimaron distintos modelos de regresión logística multinivel. En el primer modelo, la variable dependiente sufrió o no algún delito en los últimos doce meses y la variable explicativa de interés fue si realizó actividades dentro o fuera del hogar, tal como ya se explicó antes. Una vez controlado el modelo por otras variables sociodemográficas y socioeconómicas (sexo, edad, nivel de escolaridad, si posee celular) los resultados indican, en primer lugar, que es mejor colocar la variable entidad federativa en un segundo nivel y no como factor en el mismo nivel en un modelo de regresión logística. Las 


\section{Gráfica 3}

Proporción de jóvenes de 15 a 24 años según el tipo de actividad de diversión, ambos sexos

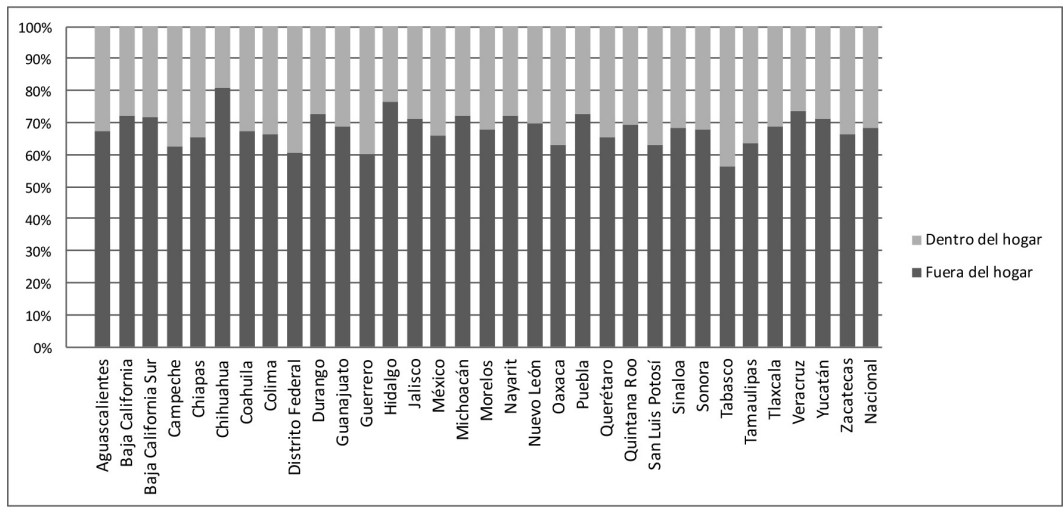

Fuente: elaboración propia con base en la Enjuve (IMJ, 2010).

\section{Gráfica 4}

Proporción de jóvenes de 15 a 24 que fue víctima de delito por tipo de actividad principal de esparcimiento, ambos sexos, porcentajes

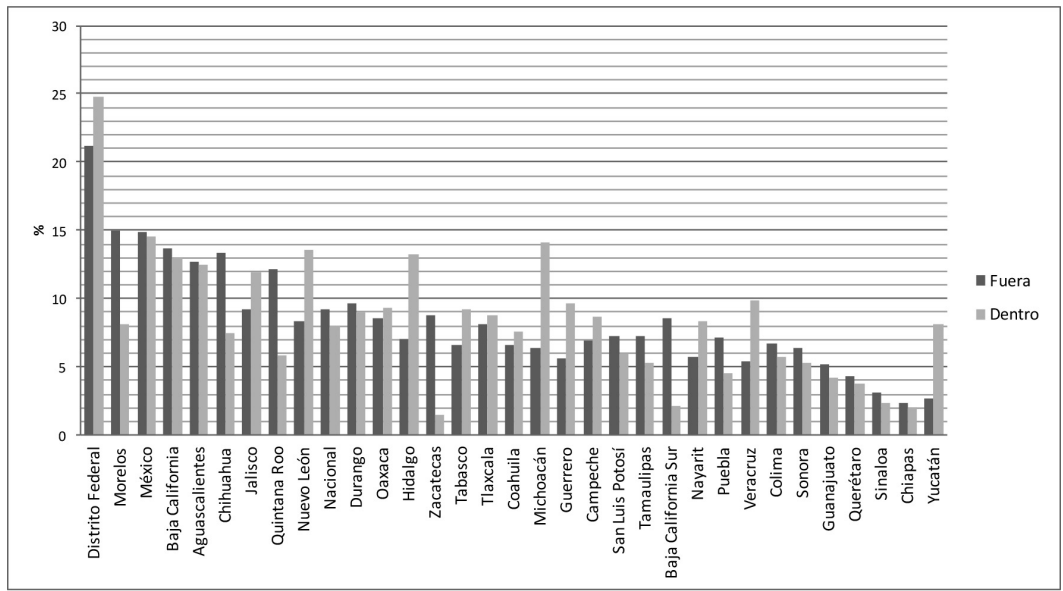

Fuente: elaboración propia con base en la Enjuve (IMJ, 2010).

razones de momios asociadas a ser hombre, tener una escolaridad de preparatoria y más, tener celular, indican un incremento en la probabilidad de haber sido víctima de delito (cuadro 3). La variable que nos interesa, actividad de diversión, no tuvo diferencias estadísticamente significativas entre las dos categorías (actividad fuera del hogar y actividad dentro del hogar). 


\section{Cuadro 3}

Modelo 1. Modelo de regresión logística multinivel sobre factores relacionados con haber sufrido delito por los jóvenes de 15 a 24 ańos, con actividad de diversión en dos categorías (fuera o dentro del hogar)

\begin{tabular}{lcc}
\hline \multicolumn{1}{c}{ Sufrió delito (No, Si) } & Odds Ratio & $P>z$ \\
Edad (continua) & 1.05 & $* * *$ \\
Sexo & & \\
Mujer & 1.00 & $* * *$ \\
Hombre & 1.47 & \\
Escolaridad & & \\
Primaria o menos & 1.00 & $* * *$ \\
Algún nivel de secundaria & 1.04 & $* * *$ \\
Algún nivel de preparatoria o equivalente & 1.30 & $*$ \\
Algún nivel de licenciatura o más & 1.69 & \\
Tenencia de celular & & \\
No & 1.00 & \\
Sí & 1.42 & \\
Principal actividad de diversión en el tiempo libre & & \\
Fuera del hogar & 1.00 & \\
Dentro del hogar & 1.07 & \\
\hline
\end{tabular}

LR test $v$ s. logistic regression: Prob $>=$ chibar $2=0.000$

*** Signif. al $0.05 \%$; *Signif. al $0.10 \%$

Fuente: elaboración propia con base en la Enjuve (IMJ, 2010).

Este resultado parece entonces ir contra las narrativas que sugieren que en contextos de alta incidencia delictiva es mejor restringir los desplazamientos y el uso de espacios fuera del hogar por parte de los jóvenes. Este primer modelo parece indicar que quienes se quedan en el hogar no parecen tener ventajas respecto a quienes realizan actividades en espacios fuera de él. En este sentido, podría afirmarse que una menor exposición de quienes se quedan en el hogar se compensaría en el caso de quienes salen del hogar con mejores habilidades para definir las condiciones de riesgo, reduciendo con ello la posibilidad de que se transformen en víctimas de un delito.

Para observar mejor cómo se comporta cada una de las actividades de esparcimiento respecto del riesgo, estimamos otros cinco modelos logísticos multinivel manteniendo desagregadas las categorías de las actividades, cambiando la actividad contra la que se comparan las demás categorías (cuadro 4). Los resultados muestran que una vez desagregadas las categorías de diversión, los efectos de edad, sexo y tenencia de celular se mantienen como en el modelo 1 . 


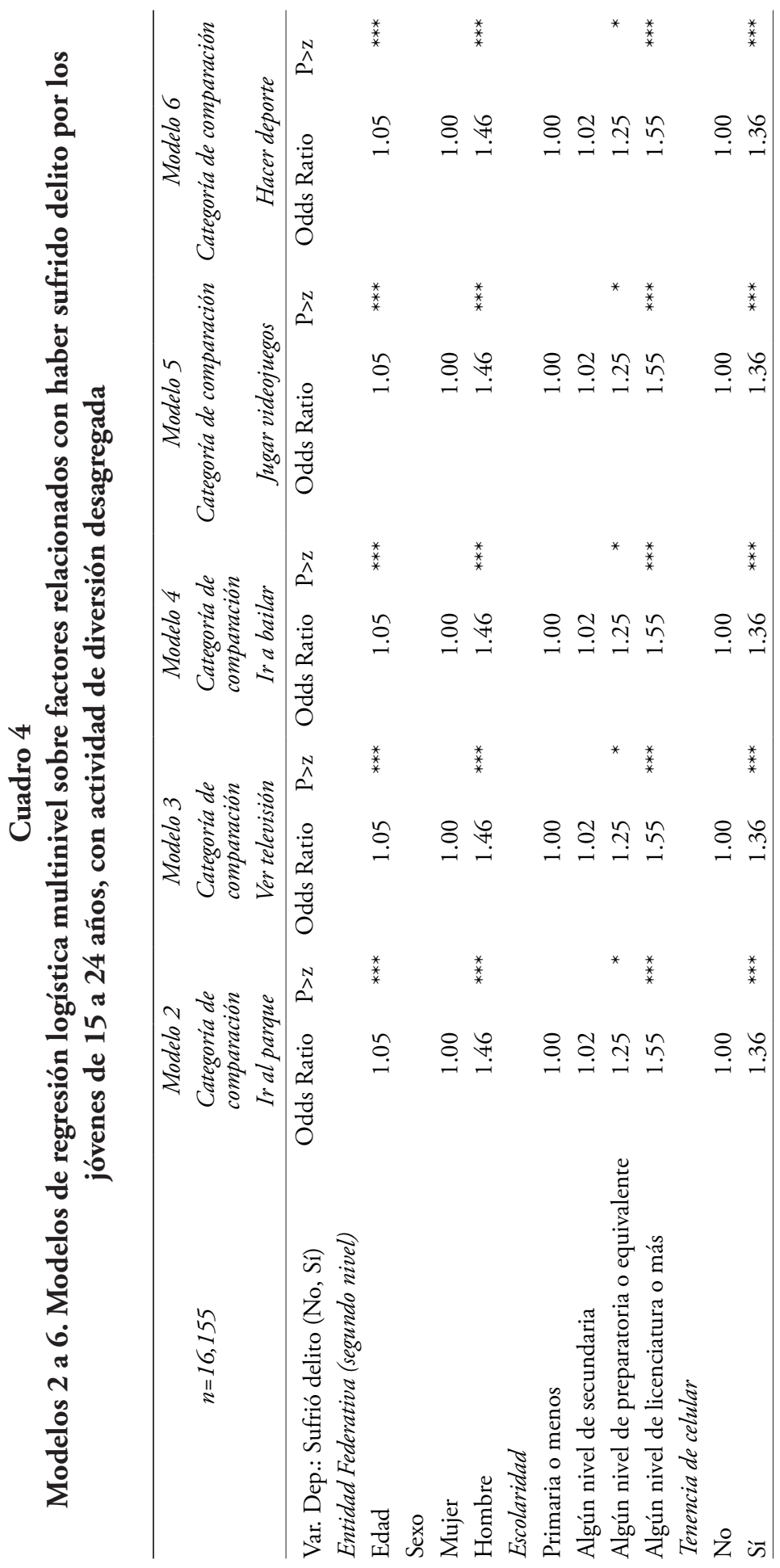




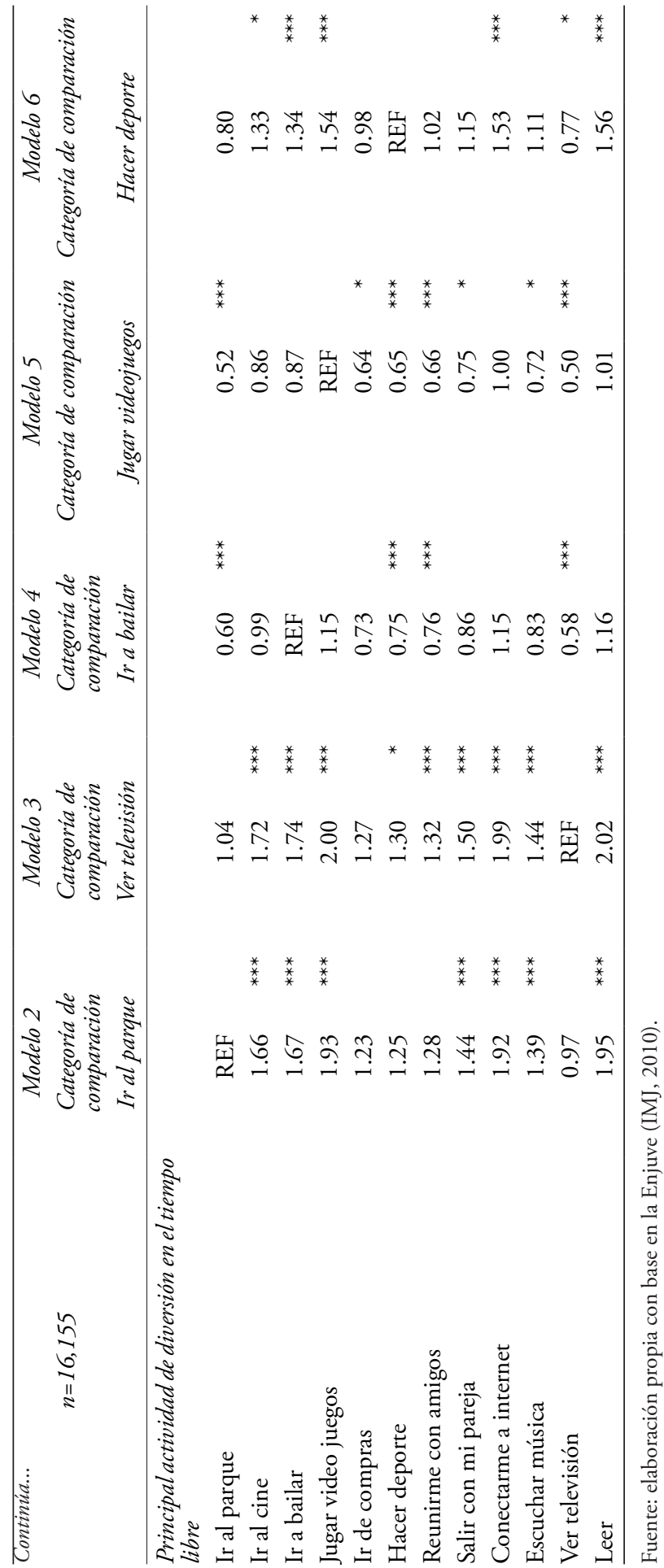


La variable escolaridad pierde la significancia estadística en la comparación entre tener algún nivel de preparatoria y más versus tener primaria o menos, y se mantiene la diferencia respecto a quienes tienen algún nivel de universidad y más en comparación con quienes tienen primaria o menos, respecto a haber sido o no víctima de delito. En cuanto a las categorías de diversión, hay tanto actividades que se realizan dentro del hogar como actividades que se realizan fuera del mismo que aparecen como de mayor riesgo para ser víctima de delito. Esto es, depende de qué tipo de actividad se realice. Jugar videojuegos y conectarse a internet son dos actividades que aparecen con el doble de momios de ser víctima de delito que otras como ir al parque, ver televisión o hacer deportes, pero no se diferencian de ir a bailar. Entre las actividades que se realizan fuera, ir a bailar también aparece en la mayoría de los modelos como una actividad con mayor riesgo, excepto cuando se la compara con jugar videojuegos. Ver televisión, en cambio, aparece en la mayoría de las comparaciones como la actividad con menores momios, lo que mostraría el carácter protector respecto a ser víctima de delito. De las actividades que se realizan fuera, ir al parque parece ser protectora, pero no se diferencia de otras como ir de compras, hacer deporte y reunirse con amigos.

En síntesis, tener mayor posibilidad de ser víctima de delito no se relaciona con realizar actividades dentro o fuera del hogar, sino que depende de las actividades específicas que se realicen tanto dentro como fuera $-y$ que podrían reflejar ciertos estilos de vida o vincularse a las capacidades para enfrentar situaciones de riesgo-, donde ver televisión e ir al parque parecen ser las más protectoras, e ir a bailar y jugar videojuegos las que conllevan más riesgos.

Este resultado rechaza la primera hipótesis, mostrando que quedarse dentro del hogar no significa tener mayor protección frente a los delitos. Sin embargo, esta relación se encuentra mediada por el sexo, la escolaridad y la posesión de un celular -es decir, variables que dan cuenta de ciertas características sociodemográficas y adscripciones socioeconómicas específicas de los jóvenes-, lo cual significa que las actividades se encuentran relacionadas con elementos vinculados con la posición que ocupa cada joven en la estructura social. Ser hombre, tener nivel escolar de universidad y poseer celular aumentan el riesgo de ser víctima de delito. Esto sugiere que estar en un estrato social más alto incrementa la posibilidad de ser víctima. ${ }^{8}$ En otras palabras, no son sólo las actividades que se realizan en el tiempo libre las que potencialmente definen los procesos de victimización, sino que tiene que ver también quiénes las realizan.

\footnotetext{
${ }^{8}$ Con la información con la que se cuenta no es posible indagar sobre las causas de la relación entre estrato social y ser víctima de delito. El modelo sólo permite establecer la existencia de la relación. Sería importante, en el futuro, explorar esta relación con otros estudios o encuestas.
} 
Así, puede resultar plausible sugerir que dos actividades que se llevan a cabo en el hogar -ver televisión y jugar videojuegos-, significan cada una de ellas una actividad protectora y de riesgo respectivamente. Como muestran otros estudios (Stein, 2010; Miethe y Drass, 1999), las actividades de ocio llevadas a cabo en la noche, como ir a los bares y a bailar, implican un incremento en el contacto entre individuos en contextos sociales donde convergen potenciales criminales y víctimas, son escenarios donde además se pueden desarrollar relaciones emocionales que se salen de control y que derivan en episodios de violencia. En el parque, por el contrario, se presentan relaciones de interacción social reguladas y procesadas por lo general fuera de contextos emocionales variables e intensos como los que se viven en bares y espacios de baile.?

Los resultados que aquí se presentan muestran que las actividades que realizan los jóvenes en su tiempo libre se asocian a los riesgos de victimización; apuntan a que no existe un patrón definido, sino que es necesario dar cuenta de la presencia de distintos factores en su conformación.

Una de las interpretaciones posibles sería que algunas actividades que se realizan fuera del hogar, como salir al parque, proporcionan capacidades para comprender el código de la calle y para conformar un contorno de seguridad. También podría pensarse que algunas actividades en el hogar posiblemente desconectan a las personas para comprender adecuadamente los códigos, tales como jugar videojuegos, u otras que funcionan en sentido contrario, como ver televisión. Lo que ambos extremos podrían representar es el peso que tiene el contexto y el estilo de vida, así como el conocimiento de los códigos de la calle para generar estrategias de protección.

\section{Conclusiones}

El presente artículo indagó, para el caso de los jóvenes mexicanos, qué tipo de actividades en el tiempo libre definen potencialmente los escenarios de victimización, al igual que el perfil de quiénes las realizan. La relevancia de un trabajo en este sentido radica en el hecho de que permitió entender una parte de los factores que contribuyen a la victimización de los jóvenes. Sobre este punto, el trabajo da indicios para estudiar la relación entre victimización y los contextos y estilos de vida de los jóvenes. Así, los jóvenes que realizan algunas actividades mostraron tener mayores riesgos de ser víctimas de delito, tal como jugar videojuegos o salir a bailar. Los hombres tienen un poco más de riesgo que las mujeres y, en los indicadores que se refieren a estrato socioeconómico (escolaridad y

\footnotetext{
${ }^{9}$ Aunque como han señalado Jorgensen et al. (2013), la mujeres tienden a manifestar un mayor sentimiento de inseguridad en los parques que los hombres, en la medida en que se sienten más vulnerables.
} 
tener celular), se muestra que el mayor nivel socioeconómico se asocia a mayor riesgo de ser víctima del delito. Estos aspectos refieren a los lugares que ellos frecuentan y las actividades que ahí realizan, y nos dicen algo sobre las capacidades que despliegan para comprender las lógicas espaciales y relacionales en donde llevan a cabo sus actividades de tiempo libre.

El trabajo no suscribe, sin embargo, una posición donde el "adentro" o el "afuera" del hogar son algo bueno o malo per se. En ambos espacios, como se pudo observar, pueden desarrollarse actividades de tiempo libre que aparentemente inhiben o fomentan la capacidad para interrelacionarse con los entornos que los exponen a la victimización. Existen ciertamente variables que influyen en dicha capacidad, tales como el sexo, el ingreso, así como otras variables más de carácter estructural. Si bien la información con la que se cuenta permite llegar a una serie de hallazgos interesantes, hace falta profundizar más en el tema. Esto permitiría contar con información clave para comprender la forma en que este sector de la población está enfrentando las condiciones de inseguridad, independientemente de las estrategias que desarrollan autoridades municipales, estatales y federales.

Así sería posible el desarrollo de políticas integrales en materia de prevención con la participación de la población victimizada. Incluso más estudios en torno al tema de victimización y uso del tiempo libre resultarían pertinentes para pensar en la construcción de espacios como bienes comunes en el sentido que Ostrom (2000) da a este término, una alternativa a los "espacios públicos-privados" o a los "espacios masivos privados", que marcan la forma en que se ha pensado la dicotomía entre el espacio público y privado. ${ }^{10}$

En un contexto de inseguridad como el que vive México, resulta cada vez más importante comprender la manera en que la sociedad pone en marcha sus capacidades de creación e innovación colectiva y personal para negociar y vivir en el miedo y la incertidumbre; cómo es que logra construir un orden social en el caos de la violencia y la inseguridad. En la medida en que se visibilicen las formas en que se hace frente al sentimiento de inseguridad desde lugares definidos de enunciación como el trabajo, la escuela, el hogar, los parques, los centros de diversión comunes y privados -y no sólo a partir de espacios tan abstractos como la colonia, el municipio o la entidad federativa- se tendrán herramientas para entender cómo

\footnotetext{
${ }^{10}$ Agradecemos a uno de los dictaminadores anónimos el haber seńalado la importancia de este tema en los comentarios a nuestro trabajo. De hecho sería importante que en una futura encuesta nacional de juventud se pudiera distinguir entre los espacios públicos -en sus múltiples dimensionesy privados, e incluir los espacios comunes, su construcción y apropiación por parte de las comunidades y en particular de los jóvenes.
} 
el aprendizaje de ciertos códigos de la calle son centrales para construir el día a día en la vida de los jóvenes.

\section{Fuentes consultadas}

Anderson, Elijah (2000), Street code: decency, violence, and the moral life of the inner city, W.W. Norton y Company, New York.

Blancas-Madrigal, Daniel (2004), “Toque de queda, así inician las dictaduras", Crónica, México, <http://www.cronica.com.mx/notas/2007/304847.html>, 6 de julio 2015.

Blokland, Talja (2008), "Facing violence: everyday risks in an American Housing Project", Sociology, 42 (4), British Sociological Association, Durham, pp. 601-617.

Caldeira, Teresa (2000), City of Walls. Crime, segregation, and citizenship in Säo Paulo, University of California Press, Los Angeles.

Camarena, Rosa María (2015), "Efectos de la delincuencia: inseguridad y miedo entre los jóvenes”, Coyuntura Demográfica, núm. 8, Sociedad Mexicana de Demografía, México, pp. 41-51.

Carmona, Matthew (2010), "Contemporary public space, part two: classification”, Journal of Urban Design, 15 (2), Institute of Urban Planning, University of Nottingham, London, pp. 157-175.

Chávez, Silvia, Israel Dávila y Javier Salinas (2004), "En marcha, toque de queda en Tlalnepantla; repudio de vecinos", La Jornada, 5 de junio, UNAM, México, <http://www.jornada.unam.mx/2004/ 06/05/030n2est.php?origen=index.html\&fly=1>, 3 de julio 2015.

Coaffe Jon, David Wood y Peter Rogers (2009), The everyday resilience of the city, Palgrave, London.

Cohen, Lawrence y Marcus Felson (1979), "Social change and crime rate trends: a routine activity approach", American Sociological Review, 44 (4), American Sociological Association, Washington, pp. 588-608.

Collins, Randall (2008), Violence. A micro-sociological theory, Princeton University Press, Princeton. 
Davis, Robert y Lucy Friedman (1985), "The emotional aftermath of crime and violence", en Charles Figley (ed.), Trauma and its Wake, Brunner-Mazel, New York, pp. 90-112.

Denkers, Adriaan y Franz Winkel (1998), “Crime victims” well-being and fear in a prospective and longitudinal study", International Review of Victimology, 5 (3-4), University of Sheffield, Thousand Oaks, pp. 93-140.

Garfinkel, Harold (1984), Studies in ethnomethodology, Polity, Cambridge.

Giddens, Anthony (1997), Modernidad e identidad del yo, Península, Barcelona.

Goffman, Erving (1986), Frame analysis. An essay of the organization of experience, Northeastern University Press, Boston.

Goffman, Erving (1977), Interaction ritual. Essay on face-to-face behavior, Pantheon Books, New York.

González, Román (2002), "El toque de queda en Tecate atropella los artículos 1 y 11 de la Carta Magna”, Cimacnoticias, 24 de mayo, México, <http://www.cimacnoticias.com.mx/node/26470>, 3 de julio 2015.

Green Eileen y Carrie Singleton (2006), "Risky bodies at leisure: young women negotiating space and place", Sociology, 40 (5), British Sociological Association, Durham, pp. 853-871.

Heitgerd Janet y Robert Bursik (1987), "Extracommunity dynamics and the ecology of delinquency", British Journal of Sociology, 94 (4), London School of Economics, London, pp. 775-787.

Hindelang, Michel, Michael Gottfredson y James Garofalo (1978), Victims of personal crime: an empirical foundation for a theory of personal victimization, Ballinger, Cambridge.

Instituto Mexicano de la Juventud (2010), Encuesta Nacional de Juventud 2010, <http://bdsocial.inmujeres.gob.mx/index.php/enjuve/17acervo/acervo/245-encuesta-nacional-de-juventud-enjuve-2010>, 3 de julio de 2015. 
Imbusch, Peter, Michel Misse y Fernando Carrión (2011), "Violence research in Latin America and the Caribbean: a literature review", International Journal of Conflict and Violence, 5 (1), University of Bielefeld, Maryland, pp. 87-154.

Jacobs, Bruce y Richard Wright (2010), "Bounded rationality, retaliation, and the spread of urban violence", Journal of Interpersonal Violence, 25 (10), University of Washington, Washington, pp. 1739-1766.

Job, Vanessa (2013) “En Zongolica, Veracruz, autoridades determinaron declarar un toque de queda para los jóvenes para disminuir los problemas de seguridad", Noticieros Televisa, 7 de febrero, México, $<$ http://noticierostelevisa.esmas.com/especiales/557794/toquequeda/>, 3 de julio de 2015 .

Jorgensen, Lisa, Gary Ellis y Eward Ruddell (2013), "Fear perceptions in public parks: interactions of environmental concealment, the presence of people recreating, and gender", Environment and Behavior, 45 (7), Connecticut College, Utah, pp. 803-820.

Jorquera, Ramón (2011), Ciudad de miedo. La seguridad y el capital social en las clases medias, Colegio de Sonora, Hermosillo.

La Policiaca (2011), "Aplican toque de queda a menores en Acuña, Coahuila”, La Policiaca, Acuña, Coahuila, 21 de abril, <http://www. lapoliciaca.com/nota-roja/aplican-toque-de-queda-a-menores-enacuna-coahuila/s, 3 de julio de 2015 .

Lupton, Deborah (1999), "Dangerous places and the unpredictable stranger: constructions of fear of crime", The Australian and New Zealand Journal of Criminology, 32 (1), Griffith University, Auckland, pp. 1-15.

Mawby, Rob y Sandra Walklate (1997), "The impact of burglary: a tale of two cities", International Review of Victimology, 4 (2), University of Sheffield, Thousand Oaks, pp. 267-296.

México Unido contra la Delincuencia (2013), Encuesta nacional sobre percepción de inseguridad ciudadana en México, Consulta Mitofsky, mayo 2013, <http://mucd.org.mx/recursos/Contenidos/ EncuestaMitofskydePercepcinCiudadanasobrela/documentos2/20130526_NA_MUCD.pdf>, 3 de julio 2015. 
Miethe, Teranse y Kriss Drass (1999), "Exploring the social contest of instrumental and expressive homicides: an application of qualitative comparative analysis", Journal of Quantitative Criminology, 15 (1), George Manson University, New York, pp. 1-21.

Nieto, Marcus, Kimberly Johnston-Dodds y Charlene Wear (2002), Public and private applications of video surveillance and biometric technologies, California Research Bureau, Sacramento.

Ostrom, Elinor (2000), El gobierno de los bienes comunes. La evolución de las instituciones de acción colectiva, UNAM-CRIM-Fondo de Cultura Económica, México.

Pérez, Verónica, David Díaz y Carmen Fernández (2014), “Percepción de la violencia social en estudiantes mexicanos de educación media, usuarios y no usuarios de sustancias", Psicología y Salud, 24 (2), Universidad Veracruzana, Xalapa, pp. 255-268.

Resick, Patricia (1987), "Psychological effects of victimization: implications for the criminal justice system", Crime \& Delinquency, 33 (1), Southern Illinois University, Cambridge, pp. 468-478.

Richards, Neil (2013), "Dangers of surveillance", Harvard Law Review, 126 (7), University of Harvard, Cambridge, pp. 1934-1964.

Salmi, Venla, Mirka Smolej y Janne Kivivuori (2007), "Crime victimization, exposure to crime news and social trust among adolescents", Young, 15 (3), University of Copenhagen, Copenhagen, pp. 255-272.

Sennett, Richard (2012), Together. The rituals, pleasures and politics of cooperation, Penguin, London.

Shapland, Joanna y Matthew Hall (2007), "What do we know about the effects of crime on victims?", International Review of Victimology, 14 (2), University of Sheffield, Thousand Oaks, pp. 175-217.

Simmel, Georg (2001), El individuo y la libertad. Ensayos de crítica a la cultura, Península, Barcelona.

Stein, Rachel (2010), "The utility of country structure: a cross-national multilevel analysis of property and violent victimology", Interna- 
tional Criminal Justice Review, 20 (1), Georgia State University, Georgia, pp. 35-55.

Sutherland, Edwin (1988), Ladrones profesionales, La Piqueta, Madrid.

Threadgold, Steven y Pam Nilan (2009), "Reflexivity of contemporary youth, risk and cultural capital", Current Sociology, 57 (1), International Sociological Association, London, pp. 47-68.

Tseloni, Aandromiche y Graham Farrell (2002), "Burglary victimization across Europe: the roles of prior victimization, micro and macrolevel routine activities", en Paul Nieuwbeerta (ed.), Crime victimization in comparative perspective, The Federation Press, Annandale, pp. 141-161.

Valenzuela, Alfonso (2013), "Power, territory, and the social control of space in Latin America”, Latin American Perspectives, 40 (2), University of California, Riverside, pp. 21-34.

Valenzuela, José Manuel (2002), "El tropel de las pasiones. Jóvenes y juventudes en México", en José Manuel Valenzuela (coord.) Jóvenes Mexicanos del Siglo XXI. Encuesta Nacional de Juventud 2000, Instituto Mexicano de la Juventud, México, pp. 27-51.

Wakefield, Alison (2005), The public surveillance functions of private security. Surveillance \& Society, 2 (4), Queen's University, Kingston, pp. 529-545.

Walklate, Sandra (2011), "Reframing criminal victimization: finding a place for vulnerability and resilience", Theoretical Criminology, 15 (2), University of Oxford, Oxford, pp. 179-194.

Walklate, Sandra (2001), "Fearful communities?", Urban Studies, 38 (5-6), Manchester Metropolitan University, New Haven, pp. 929-939.

Walklate, Sandra (1998), "Crime and community fear or trust?”, British Journal of Sociology, 94 (4), London School of Economics, London, pp. 550-569.

Wilcox, Pamela, Kenneth Land y Scott Hunt (2003), Criminal circumstance: A dynamic multicontextual criminal opportunity theory, Aldine, New York. 
Wilsem, Johan, Nan Dirk de Graff y Karin Wittebrood (2003), “Crossnational differences in victimization: disentangling the impact of composition and context", European Sociological Review, 19 (2), Oxford University Press, Oxford, pp. 125-142.

Zócalo Saltillo (2012), "Avalan padres toque de queda", Zócalo Saltillo, Coahuila, 28 de septiembre, <http://www.zocalo.com.mx/seccion/articulo/avalan-padres-el-toque-de-queda>, 3 de julio de 2015.

Recibido: 4 de febrero de 2015. Corregido: 29 de septiembre de 2015. Aceptado: 9 de diciembre de 2015.

Nelson Arteaga. Mexicano. Doctor en sociología por la Universidad de Alicante, España. Es profesor-investigador de la Facultad Latinoamericana de Ciencias Sociales, México. Miembro del Sistema Nacional de Investigadores, nivel III y de la Academia Mexicana de Ciencias. Su línea de investigación actual es violencia, vigilancia y seguridad en América Latina. Entre sus últimas publicaciones destacan: "Surveillance footage and space segregation in Mexico City", International Sociology, 30 (6), International Sociological Association, Londres, pp. 619-636 (2016); "The landscape of meaning, a metaphor in process", Czech Sociological Review, 51 (3), Sociologický ústav AV ČR, pp. $493-498$ (2015); “Securitization and urban space: the case of a skyscraper in Mexico City" en Lucas Melgaço, Georgina Varna y Francesca Menichelli (eds.), Order and Conflict in Public Space, Routledge, London, pp. 37-61 (2016).

Cecilia Gayet. Argentina. Doctora en Estudios de población por El Colegio de México. Actualmente es profesora-investigadora de la Facultad Latinoamericana de Ciencias Sociales, México. Miembro del Sistema Nacional de Investigadores, nivel II. Sus líneas de investigación son salud sexual y reproductiva y estudios sobre juventud. Entre sus últimas publicaciones destacan: Infecciones de transmisión sexual en México: una mirada desde la historia y el género, Secretaría de Salud-Censida, México (2015); "Transitions to adulthood in developing countries", Annual Review of Sociology, núm. 40, The Annual Reviews, Palo Alto, pp. 521-538 (2014); en coautoría, "Calendario de inicio sexual en México. Comparación entre encuestas nacionales y tendencias en el tiempo", Salud Pública de México, 56 (6), Instituto Nacional de Salud Pública, Cuernavaca, pp. 638-647 (2014); "Sexualidad sin matrimonio. Cambios en la primera relación sexual 
de las mujeres mexicanas durante la segunda mitad del siglo XX", en Cecilia Rabell (coord.), Los mexicanos: un balance del cambio demográfico, Fondo de Cultura Económica, pp. 350-385 (2014); en coautoría, "Sexual practices of Latin America and the Caribbean", en Amanda K. Baumle, International Handbook on the Demography of Sexuality, Springer, pp. 67-90 (2013).

Alejandro Alegría. Mexicano. Maestro en Ciencias, profesor-investigador de la Facultad Latinoamericana de Ciencias Sociales, México. Ha sido profesor titular de la Universidad Autónoma Metropolitana en la División de Ciencias Básicas e Ingeniería y profesor numerario en el Departamento de Estadística del Instituto Tecnológico Autónomo de México. Asimismo imparte diversos cursos en diplomados y en la maestría de administración de riesgos en esta última institución. Fue profesor invitado en el Departamento de Actuaría y Estadística de la Universidad de Waterloo, Ontario, Canadá. También ha sido profesor en la licenciatura de Economía del ITESM, campus México, en la Escuela de Graduados de esa misma institución, en los cursos de Verano y en el doctorado de ciencias sociales del Colegio de México y en el Programa de Estudios Superiores en Banca Central, Mención en Economía y Finanzas Cuantitativas del Banco de Guatemala. Actualmente es profesor-investigador de la Facultad Latinoamericana de Ciencias Sociales, México. Forma parte del Comité Técnico para el examen de Estadística del Ceneval. Su línea de investigación actual es el uso de estadística bayesiana en el desarrollo de modelos de población. Publicación reciente: "Restricting forecasting with a VEC model: validating the feasibility of economic targets", Estadistica, 60 (174-175), Instituto Interamericano de Estadística, Rosario, pp. 83-98 (2008). 